\title{
Description of the Dependence Strength of Two Variogram Models of a Spatial Structure Using Archimedean Copulas
}

\author{
Moumouni Diallo ${ }^{1}$, Diakarya Barro ${ }^{2}$ \\ ${ }^{1}$ FSEG, Université des SSG. BP: 2575 Bamako, République du Mali \\ ${ }^{2}$ Université Ouaga II. BP: 417 Ouagadougou 12, Burkina Faso \\ Correspondence: Moumouni Diallo, FSEG, Université des SSG. BP: 2575 Bamako, République du Mali. E-mail: moudi- \\ allo1@gmail.com
}

Received: November 22, 2016 Accepted: January 5, 2017 Online Published: January 23, 2017

doi:10.5539/jmr.v9n1p117～URL: http://dx.doi.org/10.5539/jmr.v9n1p117

\begin{abstract}
Variogram is a geostatistical tool which describes how the spatial continuity changes with a given separating distance between pairs of stations. In this paper, we study the dependence structure within a same class of bivariate spatialized archimedean copulas. Specifically, we point out properties of the gaussian variogram and the exponential one. A new measure of similarity of two copulas is computed particularly between the spatial independent copula and full dependence one.
\end{abstract}

Keywords: variogram, gaussian distribution, Archimedean copulas, similarity, exponential variogram, tail dependent coefficient

2010 MSC: $62 \mathrm{H} 20,62 \mathrm{H} 11,60 \mathrm{G} 15$

\section{Introduction}

The main objective of geostatistical analysis is the characterization of spatial phenomenos that are incompletely known. Different definitions of geostatistic have been proposed by spatial statistics researchers. Thereby, geostatistics can be defined as a branch of statistics focusing on spatial or spatio-temporal datasets. While G. Matheron (Matheron, G., 1969)found in geostatistics the application of probabilistic methods to regionalized variables, Issaks E. H. et all (Helena, F., 2012) rather defined them as a way of describing phenomenons and provides adaptation of classical regression technics to take advantages of this continuity. Geostatistics covers mainly three subdomains of statistical studies: analysis of variogram, krieging and stochastic simulation. All of these subdomains use variogram models, so that variogram lies at the earth of every geostatistical activity.

The variogram function describes the degree of spatial dependence of a given spatial random field or stochastic process $\{Z(x), x \in D\}$. This tool plays with the madogram a key role in spatial modelling, estimations and inference properties given data constraints. The simple form for madogram is for all $h \in \mathbb{R}^{d}$

$$
M_{F}(h)=\frac{1}{2} E(|F[Z(x+h)]-F[Z(x)]|), \quad x \in \mathbb{R}^{d}
$$

where $\mathrm{h}$ is the average value of the separating distance between the two points (Shepard, R. N., 1987).

Moreover, the concept of F-madogram has been introduced by Cooley et al. (Shepard, R. N., 1987) to generalized is the $\lambda$-madogram associated to the distribution underlying the stochastic process $\{Z(x)\}$.

$$
\left.\gamma_{F}(h)=\frac{1}{2} E\left\{\left|[F(Y(x))]^{\lambda}-[F(Y(x+h))]^{1-\lambda}\right|\right\} ; \lambda \in\right] 0,1[.
$$

In the same way, combining results of spatial statistics and multivariate dependence tools, Barro D. (Diakarya, B., 2012) used spatial extreme values copulas to characterize the $\lambda$-madogram of process distribution under a distortional assumption. Suppose $\mathrm{H}$ is a bivariate distribution satisfying the key assumption. If its associated multivariate EV distribution marginal are unit-Fréchet distributed, then, the $\lambda$-madogram is given by

$$
\gamma_{\lambda}(h)=\frac{1}{D_{h}(\lambda, 1-\lambda)+\lambda}-c(\lambda) \text { where } c(\lambda)=\frac{2 \lambda(1-\lambda)+1}{2(\lambda+1)(2-\lambda)} ;
$$

where $D_{h}$ is a conditional spatial measure convex defined on the unit simplex of $\mathbb{R}^{2}$. In spatial prevision in particular, the 
current problem is that one needs to replace empirical model by an theorical admissible variogram model. These models are characterized by a nugget, sill and range. In practice, for a same data, different geostatisticians can obtains different paramaters because of the their appreciations.

The main contribution of this study is to study the dependence structure between two models of variograms within a same class. A new measure of similarity of two copulas are computed in particular between the spatial independent copula and full dependence one.

\section{Prelimaries}

In this section we collect important definitions and properties on bivariate copulas, variogram tail dependence coefficient. These tools turn out to be necessary for our approach. We refer the readers to standard references for copulas analysis as Joe (1997) which provide detailed and readable introductions to copulas.

\subsection{Elements of Copulas Analysis}

Introduced in spatial analysis by authors as Blanchet(Shepard, R. N., 1987)and Kazianka (Kazianka, H. 2009)copulas functions constitutes the fundamental tool in dependence modeling in statistics.

Definition 1 (Diakarya, B., 2012) Let $X=\left(X_{1}, \ldots, X_{n}\right)$ be a random vector with multivariate continuous distribution function (c.d.f.) $\mathrm{H}$ and c.d.f marginal $H_{1}, \ldots, H_{n}$. The copula of $\mathrm{X}$ (of the c.d.f. $\mathrm{H}$ respectively) is the multivariate c.d.f. C of the random vector $U=\left[H_{1}\left(X_{1}\right), \ldots, H_{n}\left(X_{n}\right)\right]$. Due to the continuity of $\left\{H_{i}, 1 \leq i \leq n\right\}$, each component of $\mathrm{U}$ is standard uniformly distributed, i.e., $U_{i} \backsim U(0,1)$ for $i=1, \ldots, n$.

Particularly, every n-copula must satisfy the n-increasing property, that means that, for any rectangle $B=[a, b]^{n} \subseteq \mathbb{R}^{n}$, the $\mathrm{B}$-volume $C_{B}$ of $\mathrm{C}$ is positive, i.e

$$
C_{B}=\int_{B} d C(u)=\sum_{i_{1}=1}^{2} \ldots \sum_{i_{n}=1}^{2}(-1)^{i_{1}+\ldots+i_{n}} C\left(u_{1 i_{1}}, \ldots, u_{1 i_{n}}\right) \geq 0 .
$$

In a bivariate study, the relation (2.1) is equivalent to the rectangular inequality, that is, for all $\left(u_{1}, v_{1}\right) ;\left(u_{2}, v_{2}\right) \in[0,1]^{2}$ with $u_{1} \leq v_{1}$ and $u_{2} \leq v_{2}$,

$$
C\left(v_{1}, v_{2}\right)-C\left(u_{1}, v_{2}\right)-C\left(v_{1}, u_{2}\right)+C\left(u_{1}, u_{2}\right) \geq 0 .
$$

Two particular copulas are used in multivariate dependence modelling: the independent or product copula $\Pi$, defined for all by:

$$
\Pi\left(u_{1}, \ldots, u_{n}\right)=u_{1} \times \ldots \times u_{n} \text { for }\left(u_{1}, \ldots, u_{n}\right) \in[0,1]^{n}
$$

and the complete dependence one, given for a parameter $\theta$ and in bivariye case by $C_{\theta}$

$$
C_{\theta}\left(u_{1}, \ldots, u_{n}\right)= \begin{cases}M(u, v)-\theta & \text { if }(u, v) \in[\theta, 1-\theta]^{2} \\ W(u, v) & \text { otherwhere }\end{cases}
$$

where $M$ and $\mathrm{W}$ are the so called bounds of Fréchet.

Particularly, an n-dimensional copula $\mathrm{C}$ is an Archimedean copula, if there exists a continuous and strictly decreasing convex function $\phi$, the generator of $\mathrm{C}$, in the class of completely monotone functions

$$
\left\{\phi:[0,+\infty] \longrightarrow[0,1] ; \phi(0)=1 ; \phi(\infty)=0 ;(-1)^{k} \frac{\partial^{k} \phi^{-1}(t)}{\partial t^{k}} \geq 0 ; k \in \mathbb{N}\right\}
$$

with generalized inverse $\phi^{-1}(y)=\inf \{t \in[0,1], \phi(t) \leq y\}$ such that,

$$
\mathrm{C}\left(u_{1}, \ldots, u_{n}\right)=\phi^{-1}\left[\phi\left(u_{1}\right)+\ldots+\phi\left(u_{n}\right)\right] ; \text { for all }\left(u_{1}, \ldots, u_{n}\right) \in[0,1]^{n} .
$$

\subsection{Setting of Variogram}

The variogram is a measure of dissimilarity. Let $S=\left\{s_{i} \in \mathbb{R}^{d}, 1 \leq i \leq n, d=1,2,3\right\}$ be the set of $n$ spatial sites. In classic statistic the computation of the parameters is relatively possible because the repetition of independent data. However in spatial statistics one can study the most of the time a single observation in each site. Therefore, in isotropical erea one groups these sites according to their separed distance $\mathrm{h}$ and we compute the parameters of interest. In the practice, the paramaters depend on chosen step (espace unity). 
Let $Y(s)$ be the regionalized variable, then the empirical version of variogram is computed as

$$
\tilde{\gamma}(h)=\frac{1}{2 \sharp N(h)} \sum_{N(h)}[Y(s+h)-Y(s)]^{2}
$$

where $N(h)$ is the set of pair sites $\{s+h, s\}$ separated by a magnitude lag $h$ and $\sharp N(h)$ its cardinal.

Our key assumption may be assumed and strenghtened by the intrinsec hypothesis

$$
\begin{cases}\mathbb{E}[Y(s)-Y(s+h)] & =0 \\ 0.5 \mathbb{E}\left\{[Y(s)-Y(s+h)]^{2}\right\} & =\gamma(h)\end{cases}
$$

and the continuous in quadratic which mean that

$$
\lim _{h \rightarrow 0} \mathbb{E}\left([Y(s+h)-Y(s)]^{2}\right)=0 .
$$

Next, we denote $\mathcal{H}$ the set of spatial fields that respect these previous conditions.

\section{Main Results}

In this paper the main objective is to describe the strenght of the dependence between two variogram functions in the same erea.

\subsection{Concept of Class of Variograms}

In this subsection, we study the dependence structure in two main classes which are exponential class and gaussian one. The spatial structure has about the intrinsic hypothesis. Then, any admissible variogram functions $\gamma$ is supposed continuous at the origin, has a sill $\varpi$ (asymptotically or not) and concave function.

Definition 2 Two variogram functions $\gamma_{1}$ and $\gamma_{2}$ are said to be of the same type if and only if, for all $h \in[0,+\infty]$ there exists two real quantities $\beta>0, \alpha \in \mathbb{R}$ such that

$$
\gamma_{1}(h)=\beta \gamma_{2}(\alpha h) .
$$

Moreover, the set of variograms of the same type is called a class of variograms.

Endeed, it is easy to prove that the relation(9) is an equivalent relation. Each of these classes is represented by its normalized version provided respectively by equation (10) for the exponential class

$$
\gamma_{E}(h)=1-\exp (-h / a), h \geq 0, a>0
$$

and by (11) for gaussian one.

$$
\gamma_{G}(h)=\left\{\begin{array}{ll}
1-\exp \left(-h^{2} / a\right), & 0 \leq h \leq a \\
1, & h \geq a
\end{array} .\right.
$$

The main objective consists in describing the strenght dependence between two variogram functions in the same region via archimedean copulas, so that, we need to find the generator of each class.

Proposition 3 Let $\gamma$ be an normalized exponential or gaussian variogram, then function $F$ defined one $\mathbb{R}$ by

$$
F(x)= \begin{cases}\gamma(x), & 0 \leq x \\ 0 & \text { otherwise }\end{cases}
$$

is a distribution function.

proof. Let $\gamma$ be an exponential or gaussian variogram normalized. Then, on $[0,+\infty[, F$ is continuous and increasing function because $\gamma$ satisfies these properties. Morever,

$$
\lim _{x \rightarrow 0} F(x)=\gamma(0)=F(0)=0, \text { also } \lim _{x \rightarrow+\infty} F(x)=\lim _{x \rightarrow+\infty} \gamma(x)=1
$$

and $\lim _{x \rightarrow-\infty} F(x)=0$. So, it follows that $F$ is a distribution function. 
For a simplicity reason, let's use the standardized version of each class of variogram in the main assumption. There is no loss of generality in this case, this standardization does not modifie the strength spatial dependence structure. It allows to reduce all scales to one without affect the spatial dependence structure. Another advantage of this standardization is the follolling proposition.

Proposition 4 Let $\gamma$ be the standardized variogram with the main asumption, their exist $\delta \in \mathbb{R}_{+} \cup\{+\infty\}$ such that the function $\breve{F}_{s}$ defined from $\mathbb{R}$ to $\mathbb{R}$ by

$$
F_{s}(x)= \begin{cases}0, & x \leq 0 \\ \gamma_{s}(x), & 0 \leq x<\delta \\ 1, & x \geq \delta\end{cases}
$$

is a distribution function.

Proof. In the main asumption, if the spatial structure has a range then $\delta$ is 1 else $+\infty$. Suppose that the spatial structure has a range, then $F$ becomes

$$
F(x)= \begin{cases}0, & x \leq 0 \\ \gamma(x), & 0 \leq x<1 . \\ 1, & x \geq 1\end{cases}
$$

Since $\gamma$ is a continuous and increasing function and

$$
\lim _{x \rightarrow 0} F(x)=\gamma(0)=F(0)=0 \underset{x \rightarrow 1}{\operatorname{and} \lim _{x \rightarrow 1} F(x)=\gamma(1)=F(1)=1}
$$

then $F$ is continuous and increasing function. Morever, it follows that

$$
\lim _{x \rightarrow-\infty} F(x)=0 \text { and } \lim _{x \rightarrow+\infty} F(x)=1 .
$$

Therefore $F$ is a distribution function as asserted. Similarly, the case $\delta=+\infty$ has been proved.

\subsection{Spatial Archimedean Copulas of a Class of Variograms}

Let $X_{s}=\left\{\left(X_{s, 1} ; \ldots ; X_{s, n}\right), s \in S\right\}$ be a continuous stochastic random vector observed at a finite number of locations $S_{m}=$ $\left\{s_{1}, \ldots, s_{m}\right\} ; s_{i} \in \mathbb{R}^{2}$. Barro et all (2016) have introdued the defintio of spatialized generator $\phi_{s}$ of Archimedean copulas as

$$
C_{\phi_{s}}\left(u_{1}^{\check{s}} ; \ldots ; u_{n}^{\check{s}}\right)=\phi_{s}^{-1}\left[\phi_{s}\left(u_{1}^{\check{s}}\right)+\ldots+\phi_{s}\left(u_{n}^{\check{s}}\right)\right] .
$$

for a given $s \in S$ and for all $\left(u_{1}^{\check{s}} ; \ldots ; u_{n}^{\check{s}}\right) \in[0,1]^{n}$.

Spatial copulas are used for generating joint distributions with a variety of spatial dependence structure. This section deals with how to find a spatial copula knowing the variogram function of field. Let $\phi$ be a continuous and strictly decreasing function from $[0,1]$ to $[0,+\infty]$ such that $\phi(1)=0$. The pseudo-inverse of $\phi$ is the function $\phi^{[-1]}$ is given as

$$
\phi(h)=\left\{\begin{array}{ll}
\phi^{-1}(h), & 0 \leq h \leq \phi(0) \\
0, & \phi(0) \leq h
\end{array} .\right.
$$

Proposition 5 Let $\gamma$ be an admissible standardized variogram function and $F_{\gamma}$ its associated distribution function. The bivariate distribution function $C_{\gamma}$ defined from $[0,1]^{2}$ to $[0,1]$ as

$$
C_{\gamma}(u, v)=\max \left[\varphi_{\gamma}^{[-1]}\left(\varphi_{\gamma}(u)+\varphi_{\gamma}(v)\right), 0\right]
$$

is a spatial Archimedean copula, where $\varphi_{\gamma}$ is the Laplace transform of $F_{\gamma}$ defined as

$$
\varphi_{\gamma}(t)=\left\{\begin{array}{ll}
\int_{0}^{1} \exp (w t) d F_{\gamma}(w), & \text { if } \delta<+\infty \\
\int_{0}^{+\infty} \exp (w t) d F_{\gamma}(w), & \text { else }
\end{array} .\right.
$$

Proof. Previously we prove that $F_{\gamma}$ is a distribution function. And Nelson proved that, Laplace transform of any distribution function is a Archimedean copula generator. Consequently $\varphi_{\gamma}$ is a Archimedean generator. 


\subsubsection{Application}

Applying the previous proposition, we compute the generators of Archimedean copulas associated to gaussian variogram [see Appendix 1] and associated to gaussian variogram [see Appendix 2]. The results are summarized in the following table.

This table put out that the dependence structure in gaussian class and exponential class are respectively descibed by the inferior bound of Fréchet-Hoeffding and Clayton copula with parameter one.

Table 1. Spatial Archimedean Copulas with Generators in exponential and gaussian class.

\begin{tabular}{|c|c|c|c|c|}
\hline $\begin{array}{c}\text { Classes of } \\
\text { Variograms }\end{array}$ & $F_{\gamma}(h), a>$ & & $\varphi_{\gamma}(w)$ & $C_{\gamma}(u, v)$ \\
\hline Gaussian & $\left\{\begin{array}{l}1-\exp \left(-h^{2} / a\right) \\
0\end{array}\right.$ & $\begin{array}{l}h \geq 0 \\
h<0\end{array}$ & $1-\sqrt{\pi} w$ & $\max (u+v-1,0)$ \\
\hline Exponential & $\left\{\begin{array}{l}1-\exp (-h / a) \\
0\end{array}\right.$ & $\begin{array}{l}h \geq 0 \\
h<0\end{array}$ & $(a w+1)^{-1}$ & {$\left[u^{-1}+v^{-1}-1\right]^{-1}$} \\
\hline
\end{tabular}

\subsubsection{Interpretation}

Let $C_{I}$ and $C_{D}$ denote respectively the dependence and the independent copulas. On one hand, if the two variograms in a class (gaussian or exponential class) are totally independent, then the resulting copula will be independent (i.e $\left.C_{I}(u, v)=u v\right)$. That means also that using one variogram rather than another could cause an important deviation of information. On other hand, if the two variograms of gaussian (or exponential) class was totally dependent, the resulting copula is rather dependent one (i.e $C_{D}(u, v)=\min (u, v)$ ). That situation would mean that using one variogram rather than another could not affect deeply the informations. The dependence structure of our interest classes are neither that of $C_{I}$ nor $C_{D}$. Therefore, we need to know the proximity degree between our particular classes from each of $C_{I}$ nor $C_{D}$.

Visually, by the bands of levels lines forms, the figures 2.4 and 2.5 in appendix 3 , show that the associated copula of gaussian variogram class is more similar with the independent copula than the dependente copula. But this visual diagnotic does not allow to see the similarity of associated copula of exponential variogram with neither. We need to add another method for measuring the similarity between the resulting copulas and the reference copulas. That one is discussed in the following subsection.

\subsection{Similarity between the Resulting Copulas and Reference Copulas}

In this subsection we study the similarity between two copulas by their matrix. Many measures of similarity can be found in literature. Following Shepard in (Issaks, E. H., 1989), we defined the similarity between two copulas. In the following, let denote $\tilde{\mathrm{N}}$ and $\tilde{\mathrm{M}}$ respectively the set of the $\mathrm{n}$ and the $\mathrm{m}$ first naturals.

Proposition 6 Let $A=\left\{\left(a_{i j}\right) ; i \in \tilde{N}, j \in \tilde{M}\right\}$ and $B=\left\{\left(b_{i j}\right) ; i \in \tilde{N}, j \in \tilde{M}\right\}$ be two matrix of the set of matrix with $n$ rows et $m$ colons $\mathcal{M}_{n, m}(\mathbb{R})$ defined on $\mathbb{R}$. The function $s$ defined from $\left[\mathcal{M}_{n, m}(\mathbb{R})\right]^{2}$ to $[0,1]$ as

$$
s(A, B)=\exp \left(-\max _{i \in \tilde{N}, j \in \tilde{M}}\left|a_{i j}-b_{i j}\right|\right) .
$$

is similarity measure.

Proof. Since $A=\left\{\left(a_{i j}\right) ; i \in \tilde{N}, j \in \tilde{M}\right\}$ and $B=\left\{\left(b_{i j}\right) ; i \in \tilde{N}, j \in \tilde{M}\right\}$ are two elements of $\mathcal{M}_{n, m}(\mathbb{R})$, valid similarity measure obeys to four axioms [see 11].

First

$$
s(A, B)=1
$$

which is equivalent to writte that

$$
\exp \left(-\max _{i \in N, i \in M}\left|a_{i j}-b_{i j}\right|\right)=1
$$

or, in the other words

$$
a_{i j}=b_{i j} \text {, with } i \in N \text { and } i \in M .
$$

In particular that proves that $s$ obeys to the equal of self-similarity. 
Moreover, since $\left|a_{i j}-b_{i j}\right|=\left|b_{i j}-a_{i j}\right|$, then $s(A, B)=s(B, A)$, that is, the measure $s$ is symmetric.

And then $A \neq B$ means that, there exists an pair $(i, j) \in \tilde{N} \times \tilde{M}$ such as $a_{i j} \neq b_{i j}$.

Then,

$$
\exp \left(-\max _{i \in \tilde{N}, j \in \tilde{M}}\left|a_{i j}-b_{i j}\right|\right)<1 .
$$

In other word, for all $A \neq B$ we are $s(A, B)<s(A, A)$.

Then $s$ respect the minimality condition. The last criterion is that of triangle inequality.

Let $C=\left(c_{i j}\right) i \in \tilde{N}, j \in \tilde{M}$ be a third of $\mathcal{M}_{n, m}$. For all pair $(i, j) \in i \in \tilde{N} \times \tilde{M}$,

$$
\left|a_{i j}-b_{i j}\right| \leq\left|a_{i j}-c_{i j}\right|+\left|c_{i j}-b_{i j}\right| \Longleftrightarrow s(B, A) \geq s(A, C) . s(C, B)
$$

Therefore $s$ such is a measure of similarity. Next we use it to study the similarity between the matrix engender by two copulas. For simplicity, we will say that two matrix $A$ et $B$ in $\mathcal{M}_{n, m}$ are simular when $s(A, B)=1$.

Definition 7 Let $C_{1}$ and $C_{2}$ be two copulas defined from $[0,1]^{2}$ to $[0,1]$. These copulas will say simular if and only if for all pair $(n, m) \in \mathbb{N}^{*} \times \mathbb{N}^{*}$ their respective engendered matrix $M_{1}=\left(m_{i j}^{1}\right)$ with $(i, j) \in \tilde{N} \times \tilde{M}$ and $M_{2}=\left(m_{i j}^{2}\right)$ with $(i, j) \in \tilde{N} \times \tilde{M}$ such as $m_{i j}^{1}=C_{1}\left(u_{i}, v_{j}\right)$ and $m_{i j}^{2}=C_{2}\left(u_{i}, v_{j}\right)$ for all $0 \leq u_{i}, v_{j} \leq 1$, are simular.

Let remark that the couple $\left(u_{i}, v_{j}\right)$ with $0 \leq u_{i}, v_{j} \leq 1$ are the positions of the grid points discretization of $[0,1]^{2}$. Denote $C_{\gamma_{E}}$ and $C_{\gamma_{G}}$ the copulas associated to exponential variogram and gaussian variogram respectively. Likely, $C_{I}$ and $C_{D}$ are respectively the independent copula and dependent copula.

Proposition 8 Two copulas $C_{1}$ and $C_{2}$ are simular if and only if for all $0 \leq u, v \leq 1, C_{1}(u, v)$ and $C_{2}(u, v)$ are simular.

Proof. Deduce from the simularity of two copula definition.

As far as concerned the simularity between $C_{I}, C_{D}, C_{\gamma_{E}}$ and $C_{\gamma_{G}}$, we are the following proposition.

Proposition 9 For all $0 \leq u, v \leq 1$, the associated copula satisfies to the following properties.

i) $s\left(C_{\gamma_{G}}(u, v), C_{D}(u, v)\right) \leq s\left(C_{\gamma_{E}}(u, v), C_{D}(u, v)\right)$.

ii) $s\left(C_{\gamma_{G}}(u, v), C_{I}(u, v)\right) \leq s\left(C_{\gamma_{E}}(u, v), C_{I}(u, v)\right)$.

Proof. To prove proposition 9 consists in proving that, for all $0 \leq u, v \leq 1$,

$$
C_{\gamma_{G}}(u, v) \leq C_{\gamma_{E}}(u, v) \leq C_{I}(u, v) \leq C_{D}(u, v) .
$$

In fact, $C_{\gamma_{G}}$ et $C_{D}$ are Fréchet-Hoeffding bound, they are considered as universal bounds for copulas. Then for any copula, particulary $C_{\gamma_{E}}$ and $C_{I}$, we are

$$
C_{\gamma_{G}}(u, v) \leq C_{\gamma_{E}}(u, v) \leq C_{D}(u, v)
$$

and

$$
C_{\gamma_{G}}(u, v) \leq C_{I}(u, v) \leq C_{D}(u, v), 0 \leq u, v \leq 1 .
$$

Therefore, it is enough to compare $C_{\gamma_{E}}(u, v)$ and $C_{I}(u, v)$, for all $0 \leq u, v \leq 1$.

When at less one of $u$ and $v$ is zero, we are

$$
C_{\gamma_{G}}(0, v)=0=C_{\gamma_{E}}(0, v) \quad \text { and } C_{\gamma_{G}}(u, 0)=0=C_{\gamma_{E}}(u, v) .
$$

For $0<u, v \leq 1$, we obtain

$$
C_{\gamma_{E}}(u, v)=u v /(v+u-u v)
$$

Since we have

$$
v+u-u v-1=(1-v)(1-u),
$$

then, $v+u-u v \geq 1$. Therefore

$$
C_{\gamma_{E}}(u, v) \leq C_{I}(u, v)
$$


That result shows that the dependence structure in the both classes is more similar with that of described by the independent copula.

\subsection{Simulation}

The theorical results are illustrated by a similation. With statistic sofware $R$ (Shepard, R. N. 1987), we plot the similarities

$$
s\left(C_{\gamma_{H}}(u, v), C_{K}(u, v)\right) \text { with } 0 \leq u, v \leq 1 \text { and } H \in\{G, E\}
$$

and $K \in\{I, D\}$. Indeed, we discretized regularly $[0,1]$ in $n, 1 \leq n \leq 1000$ nodes.

For each value of $n$, we ploted

$$
s\left(C_{\gamma_{H}}(u, v), C_{K}(u, v) \mid n\right), 0 \leq u, v \leq 1, H \in\{G, E\} \text { and } K \in\{I, D\} .
$$

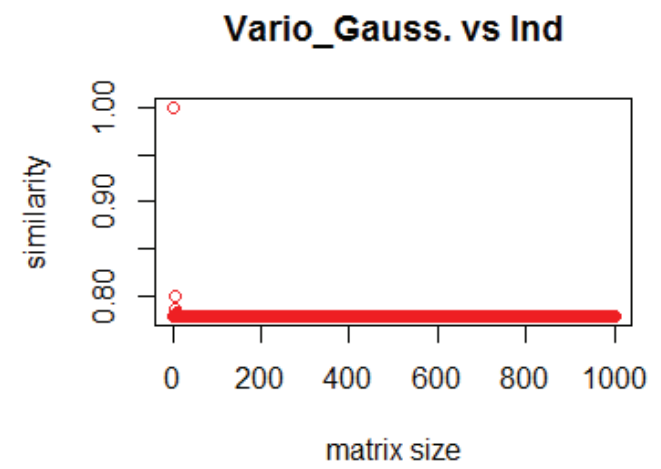

Vario_Expo. vs Ind

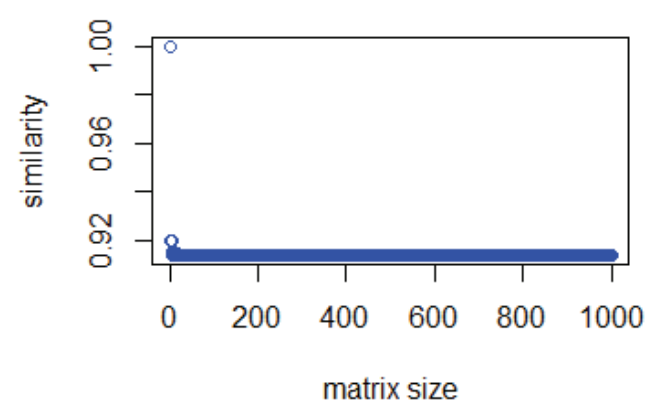

Vario_Gauss. vs Dep

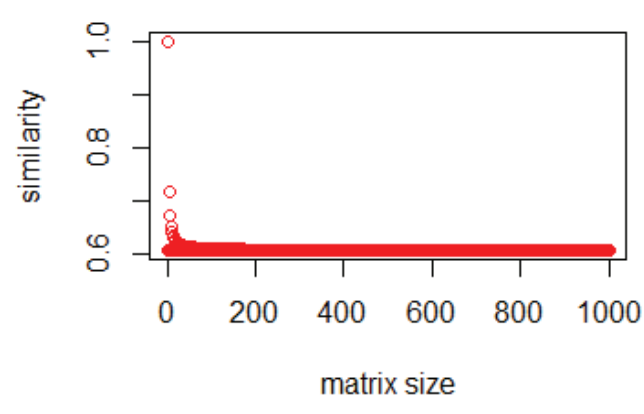

Vario_Expo.vs Dep

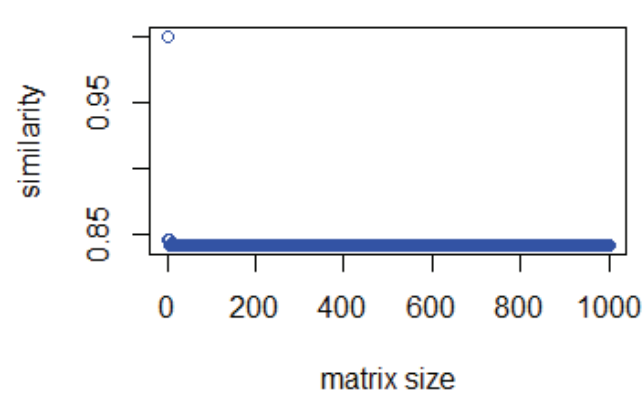

Table 2. Similarities (S. Mean) $: s\left(C_{\gamma_{G}}, C_{I}\right)$ (G.I), $s\left(C_{\gamma_{G}}, C_{D}\right)$ (G.D), $s\left(C_{\gamma_{E}}, C_{I}\right)$ (E.I), $s\left(C_{\gamma_{E}}, C_{I}\right)$ (E.I) and their standard deviations (St. D.) respectively.

\begin{tabular}{ccccc}
\cline { 2 - 5 } & G.I. & G.D. & E.I. & E.D. \\
\hline S. Mean & 0.7792889 & 0.6082728 & 0.9139695 & 0.8426776 \\
\hline St.D. & 0.009914057 & 0.018192645 & 0.003864092 & 0.007051282 \\
\hline
\end{tabular}




\subsection{Tail Dependence Coefficient of in a Class of Variograms}

Like many dependence concepts, the tail dependence coefficient describes how large (or small) values of random variables appear with large (or small) value of an other. Here, this coefficient measures the strength of link between normalized variograms in the upper-right quadrant and in the lower-left quadrant of $[0,1]^{2}$. Let $C_{\gamma}$ be the Archimedean copula associated to variogram function $\gamma$ which is either $\gamma_{G}$ or $\gamma_{E}$.

Corollary 10 Let $C_{\gamma}$ be an Archimedean copula with generator $\varphi_{\gamma}^{[-1]}$ associated to $\gamma$. Then

$$
\lambda_{U}^{\gamma}=2-\lim _{u \rightarrow 1^{-}} \frac{1-\varphi_{\gamma}\left(2 \varphi_{\gamma}^{[-1]}(u)\right)}{1-u}=2-\lim _{h \rightarrow 0^{+}} \frac{1-\varphi_{\gamma}(2 h)}{1-\varphi_{\gamma}(h)}
$$

and

$$
\lambda_{L}^{\gamma}=\lim _{u \rightarrow 0^{+}} \frac{\varphi_{\gamma}\left(2 \varphi_{\gamma}^{[-1]}(u)\right)}{u}=\lim _{h \rightarrow+\infty} \frac{\varphi_{\gamma}(2 h)}{\varphi_{\gamma}(h)}
$$

Proof. Nelson established the link between the upper tail dependence coefficient $\lambda_{U}$ and lower tail dependence coefficient with the copula $C_{\gamma}$, as

$$
\lambda_{U}=2-\lim _{u \rightarrow 1^{-}} \frac{1-C_{\gamma}(u, u)}{1-u} \quad \text { and } \quad \lambda_{L}=\lim _{u \rightarrow 0^{+}} \frac{C_{\gamma}(u, u)}{u} .
$$

The first equalities of equation (17) and (18) are shown by Nelson ( Nelson, R. B., 1999). Concerning that last one change the variables as when $u \rightarrow 1^{-}$then $h=\varphi_{\gamma}^{[-1]}(u) \rightarrow 0^{+}$(remaind that $\varphi_{\gamma}(0)=1$ )

The results of this section confirm partially those of the previous. In fact, the upper tails dependence coefficients are zero in both of cases which means that there is an asymptotical independence.

\section{Conclusion and Discussion}

Our study shows that the dependence structure in the class of gaussian variogram and exponential variogram class is more near with that of independent copula than dependent copula. Therefore, as in the theorical gaussian variogram class, two theorical exponential variograms with a too little difference can engender too important disparity in the interpretation of the spatial prediction. A new measure of similarity of two copulas are computed in particular between the spatial independent copula and full dependence one.

\section{References}

Blanchet, J. \& Davison, A. C. (2011). Spatial modelling of extreme snow depth. Annals of Applied Statistics, vol.5, N³, 1699-1725. http://dx.xoi.org/10.1214/11-AOAS464

Carlo, G. (2008). Modélisation et statistique spatial, Springer.

Cooley, D. S., Poncet, P., \& Naveau, P. (2006), Variograms for max-stable random fields. In Dependence in Probability and Statistics. Lecture Notes in Statistics 187 373-390. Springer, New York.

Diakarya, B. (2012). Analysis of stochastic spatial processes via copulas and measures of extremal dependence. Archives des Sciences, 65(12), 665-673.

Diakarya, (2016). Spatial Tail Dependence and Survival Stability in a Class of Archimedean Copulas. International Journal of Mathematics and Mathematical Sciences Volume (2016), Article ID 8927248, 8 pages http://dx.doi.org/10.1155/2016/8927248

Embrechts, P. Modelling Dependence with Copula and Applications to Risk Management, Department of Mathematics, ETHZ, CH-8092 Zürich, Switzerl.

Genest, C. (1986). Copules archimédiennes et familles des lois bidimensionnelles dont les marges sont données. The canadian Journal of Statistics, 14(2), 145-159.

Helena, F. (2012) Generalized madogram and pair wise dependence of maxima over two disjoint region of random field, Arxive prepint, arXiv:1104.2637.

Issaks, E. H. (1989), An introduction to Applied Geostatistics, Oxford university Press.

Kazianka, H. (2009). Spatial modeling \& interpolation using copulas. PhD thesis, University of Klagenfurt. Ribatet (2011)- Statistical Modelling of Spatial Extremes A. C. Davison, S. A. Padoan and M. Ribatet October 3, 2011

Matheron, G. (1969). Cours de géostatistique, Ecole des Mines de Paris. 
Nelson, R. B. (1999). An Introduction to Copulas, vol.139 of Springer Series in Statistics, springer, New York, NY,USA, 2nd edition.

Pravin, K. T. Copula Modeling: An Introduction for Practitioners, Published, sold and distributed by: now Publishers Inc.

Schmid, R. (2010). Copula-based measure of multivariate association, in Copula theory and Its applications: Proceedings of the Workshop Held in Warsaw, 25-26 September 2009, vol. 198 of lecture Notes in Statistics, 209-236, Springer, Berlin, Germany.

Shepard, R. N. (1987). Toward a universal low of generalisation for psychological science: Science,237,1317-1323.

\section{Appendice}

Appendice 1: Generator of Spatial Archimedean Copula of Gaussian Variogram

The normalized Gaussian variogram function, is written as

$$
\gamma(h)=1-\exp \left(-h^{2} / a\right), h \in \mathbb{R}_{+}, a>0 .
$$

Its associated distribution function is defined as

$$
F(h)=\left\{\begin{array}{ll}
1-\exp \left(-h^{2} / a\right), & h \geq 0 \\
0, & \text { otherwise }
\end{array} .\right.
$$

Therefore, the coresponding spatial Archimedean copula has a generator $\varphi$ defined as

$$
\begin{aligned}
\varphi(w) & =\int_{0}^{+\infty} \exp (-w t) d F(t) \\
& =\frac{\exp \left(w^{2} / 4\right)}{a} \int_{0}^{+\infty} 2 t \exp \left[-(t / a+w / 2)^{2}\right] d t
\end{aligned}
$$

After variable changing $x=t / a+w / 2$, we get

$$
\varphi(w)=\exp \left(w^{2} / 4\right)\left[\exp \left(-w^{2} / 4\right)-w \int_{w / 2}^{+\infty} \exp \left(-x^{2}\right) d x\right] .
$$

Let $I$ be

$$
\begin{aligned}
I(w) & =\int_{w / 2}^{+\infty} \exp \left(-x^{2}\right) d x \Longrightarrow I^{2}(w)=\left[\int_{w / 2}^{+\infty} \exp \left(-y^{2}\right) d y\right]\left[\int_{w / 2}^{+\infty} \exp \left(-x^{2}\right) d x\right] \\
I^{2}(w) & =\iint_{w / 2}^{+\infty} \exp \left(-x^{2}-y^{2}\right) d x d y .
\end{aligned}
$$

After a polar changing, we obtain

$$
I(w)=\sqrt{\pi} \exp \left(-w^{2} / 4\right) .
$$

Putting result (20) in the equation (19), we find the generator $\varphi(w)=1-\sqrt{\pi} w$.

Appendice 2: Generator of Spatial Archimedean Copula of Exponential Variogram

The normalized Exponential variogram function, is written as

$$
\gamma(h)=1-\exp (-h / a), h \in \mathbb{R}_{+}, a>0
$$

Then its associated distribution function is as

$$
G(h)=\left\{\begin{array}{ll}
1-\exp (-h / a), & h \geq 0 \\
0, & \text { otherwise }
\end{array} .\right.
$$

And then, the coresponding spatial archimedean copula has a generator $\psi$ defined as 


$$
\begin{aligned}
\psi(w) & =\frac{1}{a} \int_{0}^{+\infty} \exp [-(w+1 / a) t] d t \\
& =(a w+1)^{-1}
\end{aligned}
$$

\section{Appendice 3: The Figures}

In the Figure 2.4 and Figure 2.5, the levels lines are described colored bands. The colors goes from blue (mean level zero) two black (mean level one).
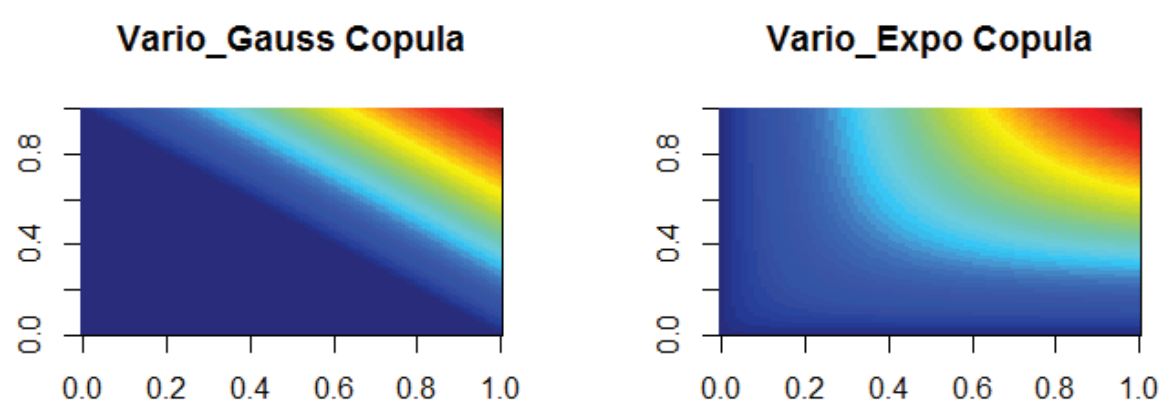

Figure 1. Images contour of: the Gaussian variogram class associated copula and Exponential variogram class associated copula

Independent Copula

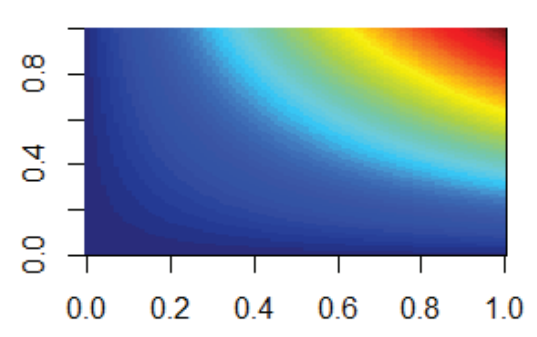

\section{dependente Copula}

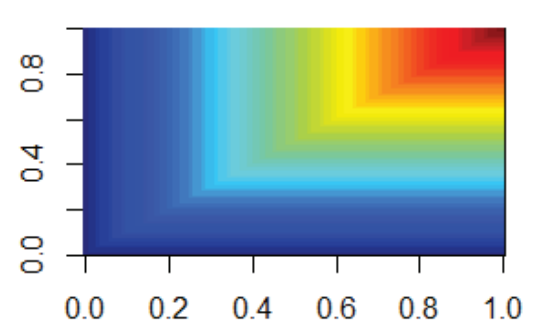

Figure 2. Images contour of Independent copula (left) and Dependent copula (rigth).

\section{Copyrights}

Copyright for this article is retained by the author(s), with first publication rights granted to the journal.

This is an open-access article distributed under the terms and conditions of the Creative Commons Attribution license (http://creativecommons.org/licenses/by/4.0/). 